\title{
Relationship between maximum bite force and the gonial angle in crossbite
}

\author{
Angelica da Veiga Said ${ }^{1}$, Patrícia Barbarini Takaki², Marilena Manno Vieira ${ }^{3}$ and Silvana Bommarito ${ }^{4 *}$ \\ ${ }^{1}$ Dental Surgeon; Master's Student, Department of Speech Pathology, Universidade Federal de São Paulo - UNIFESP/EPM, São Paulo, Brazil \\ ${ }^{2}$ Speech Therapist; Master's Student, Department of Speech Pathology, Universidade Federal de São Paulo - UNIFESP/EPM, São Paulo, Brazil \\ ${ }^{3}$ Dental Surgeon - Orthodontist; Associate Professor, Department of Speech Pathology, Universidade Federal de São Paulo - UNIFESP/EPM, São Paulo, Brazil \\ ${ }^{4}$ Speech therapist; Associate professor, Department of Speech Pathology, Universidade Federal de São Paulo - UNIFESP/EPM, São Paulo, Brazil
}

\begin{abstract}
Introduction: Modification of overjet and posterior crossbite (PC) can affect the maximum bite force (MBF), in turn affecting the shape of the gonial angle (GA).

Aims: To analyze MBF in PC and the correlation between MBF and GA in adults.

Method: The evaluation was performed in 160 individuals, i.e., 88 men and 72 women (aged 18 to 39 years), divided into two groups: without PC, comprising 130 individuals ( 75 men and 55 women) and with PC, comprising 30 individuals ( 13 men and 17 women). The method consisted of the following steps: anamnesis, evaluation of the occlusion, MBF evaluation, and GA measurement. Evaluation of the occlusion was performed in order to classify the occlusion as with or without PC. The MBF was measured with a digital dynamometer [DDK/M model (Kratos, São Paulo, Brazil)]. Three measurements were performed in all individuals with the device positioned on the occlusal surface of the first molars, alternating with one-minute intervals, following which the means were calculated. GA measurement was performed with a goniometer on both, the right and left sides. The data collected were analyzed statistically by parametric tests, considering a significance level of $0.05(5 \%)$.
\end{abstract}

Results: MBF was higher for men, independent of the group and the side; in the group without PC, MBF was different between the sides just for women. Between the groups, MBF was higher for men in the group without PC. The correlation found between MBF and GA in both groups was negative or inversely proportional.

Conclusions: The group with PC showed lower MBF in men and the correlation between MBF and GA was negative, suggesting that higher the MBF, the lower is the GA or vice versa.

\section{Introduction}

Posterior crossbite (PC) is an alteration of the occlusion in the transverse plane, characterized by a modified overjet, observed when the lower teeth protrude beyond the upper teeth [1]. This failure of the two dental arches to occlude normally in the transverse direction can result from anomalous tooth position, alveolar growth, skeletal discrepancy between the mandible and maxilla, and can be associated with asymmetric muscular function. Since spontaneous correction is rare [2], it is believed that the PC is transferred to the permanent dentition, with long-term effects on the growth and development of the stomatognathic system. According to Nakata et al. [3], individuals with this type of malocclusion may show qualitative differences in the functioning of the masticatory muscles, when compared with individuals without malocclusion, since it represents deformity in the bone bases or occlusal problems.

Maximum bite force (MBF) is a useful indicator of the functional state of the masticatory system, thereby enabling effective detection of possible masticatory alterations. Studies in children have demonstrated less electromyographic activity on the cross side during mastication [4], muscle atrophy visualized by ultrasonography (US), and decreased MBF [5].

Biomechanical forces produced during mastication are considered a primary agent for stimulating the craniofacial growth and development. One of the structures that are involved in this process is the gonial angle (GA).

GA is an angle formed by the junction of two imaginary lines that are tangential to the lower border of the mandibular body and the posterior border of the mandibular ramus. It is commonly used to determine the mandibular rotation, and is used in the diagnosis of the patient's growth pattern. In addition, the GA shape is directly related to the function of the mandibular elevator muscles [6].

Thus, if the presence of MBF can compromise the masticatory function demonstrated by reduced bite force, and the shape of the GA is related to the function of the mandibular elevator muscles, we can thus hypothesize that in the presence of this malocclusion, decreased $\mathrm{MBF}$ or asymmetric muscular function and consequently, more open or asymmetric GA are present. Furthermore, the limited evidence in adults requires studies to verify if the functional alterations found in children maintain with negative effects during growth and development

Correspondence to: Silvana Bommarito, Speech therapist; Associate professor, Department of Speech Pathology, Universidade Federal de São Paulo UNIFESP/EPM, São Paulo, Brazil, E-mail: bomarito@terra.com.br

Key words: bite force, mandible, malocclusion

Received: May 11, 2017; Accepted: May 26, 2017; Published: May 29, 2017 
of the stomatognathic system, or if they are adapted in the adult phase. Therefore, the aim of this study was to analyze the MBF in unilateral PC and the correlation between MBF and GA in adults.

\section{Materials and methods}

We performed an analytical, observational, and transverse study with the approval of the Ethics in Research Committee of the Federal University of São Paulo - UNIFESP, number 733.055. All the participants agreed to and signed the free and informed consent form (FICF).

\section{Sample description}

In this study, a sample of 160 individuals (88 men and 72 women, aged 18 to 39 years) was divided into two groups: without PC (130 individuals [75 men and 55 women]) and with PC (30 individuals [13 men and 17 women]). The study participants were students and volunteers from UNIFESP, University of São Paulo, Guarulhos, Osasco, São José dos Campos, and Baixada Santista; and soldiers enlisted in the $2^{\text {nd }}$ Brazilian Army Police Battalion, based in Osasco city.

The inclusion criteria were the presence of complete dentition i.e., at least 14 teeth in each dental arch, from the right to the left second molars, normal occlusion or Angle's Class I malocclusion. In the group with PC, the inclusion criteria also comprised of the presence of at least one posterior tooth in a crossed position, on any side.

The exclusion criteria for both groups were dental loss, presence of syndromes and craniofacial malformations, neurologic and/ or psychiatric diseases, rheumatic diseases, orthodontic treatment in progress or finished less than three years ago, complaints of parafunctional habits, and symptoms of temporomandibular disorder (TMD).

\section{Procedure}

Evaluation of the participants in this study consisted of the following steps:
A. Anamnesis,
B. Occlusion evaluation,
C. MBF measurement, and
D. GA measurement.

Data were collected at the Orofacial Motricity Outpatient Clinic at the São Paulo campus of the UNIFESP by a trained orthodontist. Collected data were entered Microsoft Excel $^{\circledR}$ spreadsheets. The evaluation was carried out in steps described below.

Anamnesis: Anamnesis was performed with the individual to obtain personal identification data (name, date of birth, age, sex, telephone number, city, and e-mail), and general and dental health histories.

Occlusion evaluation: An orthodontist evaluated occlusion by direct observation. All the individuals were seated, with the feet resting on the ground, forming 90 degrees' angles between the thighs and the legs and between the thighs and the upper body. The head was positioned with the Frankfort plane parallel to the ground and the median sagittal plane perpendicular to the ground. The occlusion was observed with the teeth in maximum intercuspation.

Using the criteria adopted for sample selection, all the individuals had normal occlusion or Angle's Class I malocclusion. It was used for the evaluation of overbite, with a limit of $1 / 3$ of the height of the clinical crown of the lower incisors. The overjet was limited at three millimeters horizontal overlap between the upper and lower anterior teeth. In the group with PC, all the participants were required to have alteration of at least one posterior tooth in the transverse (overjet) plane, and unilaterally, in a crossed position.

MBF measurement: MBF was measured using a digital dynamometer, DDK/M model (Kratos, São Paulo, Brazil). This device was developed exclusively to determine the force applied by an individual while biting. It has scales in kilogram-force (kgf), poundforce (lbf), and Newton (N); and options for dynamic measurements of the maximum force (peak). For this study, the device was configured for measuring the peak force in Newton. For biosafety purpose, the dynamometer was sanitized with $70 \%$ alcohol and its bite region was protected with a transparent plastic wrap.

With the individual sitting as described above, the dynamometer was placed between the upper and lower first molars of the right and left sides, alternatively. The measurements were started from the right side of the individual. The patient was instructed to bite on the sensor of the device as strongly as possible. Each volunteer bit the device three times on each side, alternating with one-minute intervals. Then, the average of the three measurements from each side was calculated.

GA measurement: GA was measured with the goniometer directly on the face of the individual. This device is composed of two hinged rulers at one of its ends, such that they determine the angle formed between them, when not overlapping. For evaluation, the individual was seated as described above. For biosafety purpose, the goniometer was sanitized with $70 \%$ alcohol after each use.

GA evaluation was performed in two steps: localization and measurement. For GA localization, the head of the individual was bent laterally and the index fingers were used to palpate the lower margin of the body and the posterior ascending ramus of the mandible, their junction being the GA. For measurement, the goniometer was positioned with its stems tangential to the lower margin of the mandibular body and the posterior ramus of the mandible (Figure 1).

\section{Statistical method}

Collected data were statistically analyzed using analysis of variance (ANOVA) test, Student's paired t test, and Pearson correlation, considering a significance level of 0.05 (5\%). The intensity of the correlation was given according to the table below:

Insignificant Poor Regular Good Great

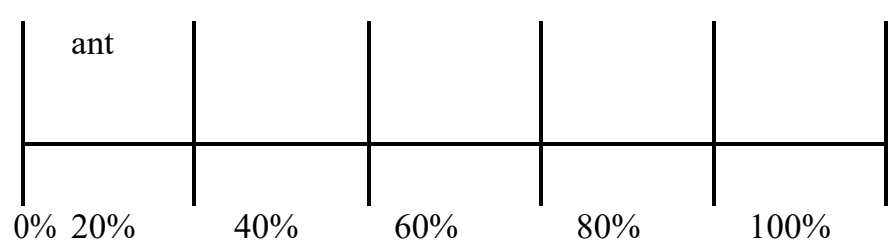

Statistically significant results were marked with an asterisk.

\section{Results}

In the groups without and with $\mathrm{PC}$, the MBF means were higher for males $(620.9 \mathrm{~N}$ and $527 \mathrm{~N}$, respectively) than females (401.7 N and $380.8 \mathrm{~N}$, respectively), independent of the right or left side. Comparing MBF between the sides, a statistically significant difference was 


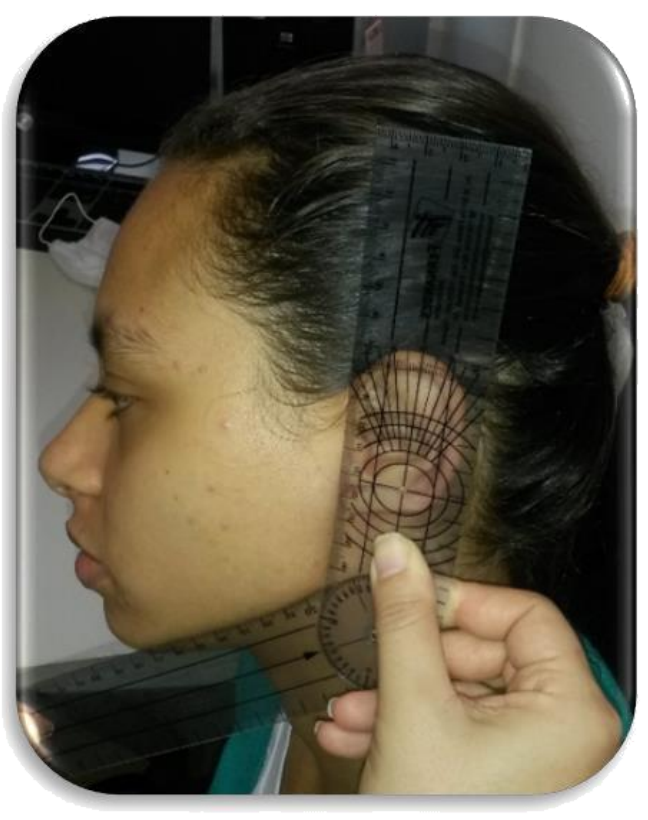

Figure 1. Goniometer in position

observed only among women in the group without PC, with values of $386.7 \mathrm{~N}$ for the right side and $416.7 \mathrm{~N}$ for the left side.

Comparing MBF means between the groups without and with PC, statistically significant differences were found in males, with higher means in the group without PC. When the groups without and with PC were analyzed, we found statistically significant differences between the groups regardless of sex, with the highest means in the group without PC (Table 1).

Considering GA, the means between the sexes and sides were similar in both the groups.

The correlations observed between MBF and GA in both the groups were negative, or inversely proportional, indicating that higher the $\mathrm{MBF}$, the lower is GA or vice versa. Statistical significance was observed among men in the group without PC and among women in the group with PC. However, despite the existing significance, the correlations were classified as poor (-23.6\%) among men in the group without PC and regular (-47.3\%) among women in the group with PC (Table 2).

\section{Discussion}

Mastication is the main function of the stomatognathic system and when performed properly, it favors the growth and development of craniofacial structures. Evaluation of the MBF can help to understand the amount of energy developed by the elevator muscles of the mandible during this function. A good condition of the dental occlusion is necessary to obtain an efficient masticatory force, and the presence of altered occlusal relationships can decrease the strength of the masticatory muscles, consequently leading to problems such as difficulty in grinding food, reduction of muscle strength for ejection of the food bolus during swallowing, inadequate respiration due to altered position of the lips, and changes in speech (phonemic distortions). MBF measurement can be performed with fair ease; however, as the MBF levels can vary according to the method, sex, and age, it is important that the measurements are compared with appropriate reference values.
When analyzing the MBF results, we found that men showed higher MBF compared to women, regardless of the side, and these findings are in agreement with studies by Bonakdarchian, Askari and Askari [7], Palinkas et al. [8], Koç, Dogan and Bek [9], de Abreu et al. [10], Takaki, Vieira and Bommarito [11], and Roldán et al. [12].

In the study by Bonakdarchian, Askari and Askari [7], performed in individuals with similar age average as our sample, we found MBF means of $721.7 \mathrm{~N}$ for men and $519.7 \mathrm{~N}$ for women (values converted to $\mathrm{N}$, since the original measurements were in $\mathrm{kgf}$ ) while our results were lower. The difference can be justified by the divergence of the method used in our study. The device used by them showed a height of $8.6 \mathrm{~mm}$ and the MBF used was the highest value among the three measurements, while our device showed a height of $10 \mathrm{~mm}$ and the $\mathrm{MBF}$ used was the average of the three measurements. Our findings are also close to those obtained by Abreu et al. [10], measured by the same device that was used by our team, with mean MBF of $630 \mathrm{~N}$ for men and $424 \mathrm{~N}$ for women.

In the study by Palinkas et al. [8], where the same device was used, the authors considered $\mathrm{MBF}$ as the highest force obtained in three measurements, in a group with similar ages to our sample. The study reported lower values, with means of $339 \mathrm{~N}$ on the right side and $348 \mathrm{~N}$ on the left side for men, and $221 \mathrm{~N}$ on the right side and $247 \mathrm{~N}$ on the left side for women. Regardless of the side, these values represented about $30 \%$ higher MBF in men than in women, while our results showed $54.56 \%$ higher MBF in men. Takaki et al. [11] used the same method as this study; however, for different age groups. MBF was $12.21 \%$ higher in men when compared to women, suggesting the influence of sex and age in MBF.

Our study also demonstrated higher MBF values compared to values measured by Koç et al. [9], obtained from teenagers aged 19 to 20 years. Using a different method from ours, with bilateral and simultaneous measurement and considering the mean of three measurements as $\mathrm{MBF}$, the study reported values of $349.1 \mathrm{~N}$ for men and $276.5 \mathrm{~N}$ for women. Van der Bilt et al. [13] also performed a study with bilateral and simultaneous measurement of the MBF and compared separate measurements on each side. In this case, the bilateral MBF used was the sum of the individual measurements, and was $30 \%$ higher than the values on each side i.e., $430 \mathrm{~N}$ and $429 \mathrm{~N}$ for the right and left sides, respectively.

Our study showed statistically significant differences between the $\mathrm{MBF}$ for each side only in women in the group without PC. These findings are not in agreement with Bonakdarchian et al. [7], Palinkas et al. [8], de Abreu et al. [10], which did not find any significant difference between the right and left sides in both sexes, in individuals with absence of sagittal alterations.

When we searched for crossbite, the focus of this study, we found many studies in children with proved functional alteration, exemplified by the reduced bite force [5,14-18]; however, limited evidence has been found in adults for this specific malocclusion.

Comparing between the normal and crossed sides in the group with PC, we did not find statistically significant difference between the sexes. Although Sonnesen et al. [18], and Sonnesen [17] studied children with unilateral PC with ages between 7 and 13 and between 7 and 11 years, respectively, they did not find statistically significant difference between the NL and CR sides. In the study by Choi et al. [19], the authors did not describe different MBF values between the sides, nor specified if the cross bites were unilateral, bilateral, or both. In their results, despite the evidence showing difference in MBF between men and women [7-12] 
Table 1. Comparison of MBF means between the groups without and with PC for males and females.

\begin{tabular}{|c|c|c|c|c|c|c|c|c|c|c|}
\hline & \multirow{2}{*}{ MBF } & \multirow{2}{*}{ Mean } & \multirow{2}{*}{ Median } & Standard & \multirow{2}{*}{$\mathrm{CV} / \%$} & \multirow{2}{*}{ Min } & \multirow{2}{*}{$\operatorname{Max}$} & \multirow{2}{*}{$\mathbf{N}$} & \multirow{2}{*}{ CI } & \multirow{2}{*}{ P-value } \\
\hline & & & & Deviation & & & & & & \\
\hline \multirow{2}{*}{ Females } & Without PC & 401,7 & 392,0 & 135,0 & 34 & 154,5 & 797,7 & 110 & 25,2 & \multirow{2}{*}{0,527} \\
\hline & With PC & 384,2 & 373,7 & 157,7 & 41 & 113,1 & 707,6 & 34 & 53,0 & \\
\hline \multirow{2}{*}{ Males } & Without PC & 620,9 & 659,5 & 191,7 & 31 & 156,3 & 974,0 & 150 & 30,7 & \multirow{2}{*}{$0,028^{*}$} \\
\hline & With PC & 530,7 & 546,9 & 195,7 & 37 & 229,0 & 931,8 & 26 & 75,2 & \\
\hline \multirow{2}{*}{ Both } & Without PC & 528,1 & 518,7 & 201,4 & 38 & 154,5 & 974,0 & 260 & 24,5 & \multirow{2}{*}{$0,005^{*}$} \\
\hline & With PC & 447,6 & 411,4 & 188,4 & 42 & 113,1 & 931,8 & 60 & 47,7 & \\
\hline
\end{tabular}

Legend:CV: Coefficient of variation CI - Confidence interval, * $\mathrm{p}<0.05$ (ANOVA)

Table 2. Correlation between MBF and GA in the groups without or with PC for males and females.

\begin{tabular}{|c|c|c|c|}
\hline \multicolumn{2}{|c|}{ MBF vs. GA } & Without & \multirow{2}{*}{ With PC } \\
\cline { 2 - 4 } & Corr (r) & $-8,3 \%$ & $-47,3 \%$ \\
\hline \multirow{2}{*}{ Females } & P-value & 0,390 & $0,005^{*}$ \\
\cline { 2 - 4 } & Corr (r) & $-23,6 \%$ & $-34,2 \%$ \\
\hline \multirow{2}{*}{ Males } & P-value & $0,004^{*}$ & 0,088 \\
\hline
\end{tabular}

Legend: Corr (r): Correlation

$* \mathrm{p}<0.05$ (Pearson correlation)

similar to our study, the total means involving both sexes in each group were considered. The crossbite group had MBF of $475 \mathrm{~N}$, similar to the value observed in our study, i.e., $447.6 \mathrm{~N}$, when considering both the sexes. The group without PC showed a mean MBF of $528.1 \mathrm{~N}$ for both sexes, while the normal occlusion group from Choi et al. [19] showed a mean $\mathrm{MBF}$ of $772 \mathrm{~N}$. The difference between MBF for without and with PC groups, considering the total sample, was statistically significant in Choi et al. [19] and our study.

Choi et al. [19] considered the presence of at least two molars in cross bites as the inclusion criteria for the crossbite group. Since the MBF measurement is always performed on the first molars, the participation of this tooth in malocclusion is justified and comprehensible. In our study, this particularity could not be considered due to the small proportion of the sample that fulfilled this criterion. The presence of only one posterior tooth in crossbite was sufficient for inclusion in the group with PC. However, we understand that this may have been a limitation of our study.

Some dimensions of the facial skeleton including the GA, are related to the activity of the muscles of mastication. According to Yanikoglu and Ylmaz [20], the reduced function of the masseter and temporal muscles can affect the angle of the mandible and therefore, establishing a relation between the angle conformation and MBF is inevitable. Since the masseter and medial pterygoid muscles are inserted in the angle region, the contraction strength of these muscles can also influence its configuration. The study by Rando, Hillson, and Antoine [21] does not consider MBF itself, suggesting an association between processed, softer diet of the Post-Industrial Revolution and wider angles of the mandibles, as compared to the medieval mandibles. The diet in question would require less work by the masticatory muscles, and thus, less biomechanical stimuli would be imputed to the facial skeleton, contributing to a wider conformation of the GAs of mandibles from the recent period. In contrast, Yoon and Chung [22] observed a decrease in GAs for teenagers, suggesting that the maturation and development of the masticatory muscles actively interfere with the anatomy of the angle.

In relation to this morphologic characteristic, two measurement methods proved to be consolidated and reliable. Evidence shows that irrespective of whether GA measurements are obtained by lateral teleradiography or panoramic radiography, the results are compatible [23-25]. However, no records of GA measurements directly on the face of the individual, as in the method adopted by our study, were found. The feasibility and simplicity of performing an objective evaluation, directly on the face of the individual, aroused great interest, since we need specific information like the face type, GA size, and relation between face width and the GA, to obtain better diagnosis and myofunctional prognosis.

This simplified practice without auxiliary exams would be of great value to the professional in the clinic routine, since this angle can indicate a pronounced altered overbite, as observed by El-Dawlatly et al. [26], who concluded that a reduced GA was the component that chiefly contributed to a deep bite. Furthermore, according to Antonarakis et al. [27], it can also present an indication of the stability in the correction of Class II division 1 . These patients, who experienced relapse after treatment, obtained lower MBF and wider GA in the pretreatment phase compared to a group without relapses.

In our study, when we analyzed GA averages in males and females between the groups without and with PC, we found similar averages between the sexes in both groups. Although we did not find similar studies related with age and occlusion relationships in the literature, we verified that in the study by Oksayan et al. [23], which was performed in individuals with an average age of 17 years, and in that by ZangoueiBooshehri et al. [25], with children aged 6 to 12 years, no differences in GA values were found between the sexes, when both evaluated three sagittal relationships. In contrast from these findings, Xie et al. [28] found statistically significant differences for angle values between men and women, in groups with average age of 27 and 64 years. Joo et al. [29] found wider angles in elderly women.

Comparing GA averages, between the right and left sides for men and women, in without and with PC groups, we did not find statistically significant difference between sides. Despite analyzing the GA through radiographies, our findings agreed with those presented by Oksayan et al. [23], Zangouei-Booshehri et al. [25] and Joo et al. [29] that did not find differences between the right and the left sides. In the study by Yanikoglu and Yilmaz [20], differences in angle values between the right and left sides in patients from 40 to 70 years of age were reported; however, it is worth mentioning that this patient sample had periodontal impairment.

Comparing GA averages between the groups without and with PC, we found similar averages $\left(122.1^{\circ}\right)$. We did not find studies that performed GA evaluation directly on the face in the literature; however, in studies where the measurements were performed on radiographies, the values were 124.17 [24], 123 [23] and 127.07 [25] degrees. The comparison between GA values obtained by direct measurement or radiographies is not the purpose of this study; however, the proximity found is an important finding, since it gives credibility to the direct measurement on the face. 
Thus, in the analysis of the MBF and GA in the groups without and with PC for men and women, we found negative correlations between $\mathrm{MBF}$ and GA for both groups, in other words, inversely proportionality indicating that higher the MBF, the lower is GA or vice-versa. These findings are discussed by Gregor, Hietschold, and Harzer [30], who performed a study using Magnetic Resonance and found that the volume of the masseter muscle was inversely proportional to the GA size. From this data, it is possible to infer that the higher MBF, the narrower is the GA. We also verified in our study, and classified the significant correlations as bad (-23.6\%) among men in the group without PC and regular (-47.3\%) among women in the group with PC.

We believe that the divergence of methods to obtain GA described in the literature was a limiting factor in our study. Therefore, all comparisons should be analyzed with caution.

\section{Conclusions}

The group with PC showed lower MBF for males and the correlation between MBF and GA was negative for females, indicating that higher the MBF, the lower is the GA or vice-versa.

\section{Acknowledgments}

We thank to the Postgraduate Department in Human Communication Disorders of the Federal University of São Paulo for funding part of this research.

\section{References}

1. Vieira MM (2014) Avaliação da Face: Prosopometria. São Paulo: Atheneu.

2. Kutin G, Hawes RR (1969) Posterior cross-bites in the deciduous and mixed dentitions. Am J Orthod 56: 491-504. [crossref]

3. Nakata Y, Ueda HM, Kato M, Tabe H, Shikata-wakisaka N, Matsumoto E (2007) Change in stomatognathic function induced by orthognathic surgery in patients with mandibular prognathism. J Oral Maxillofac Surg 65: 444-451. [crossref]

4. Alarcón JA, Martín C, Palma JC (2000) Effect of unilateral posterior crossbite on the electromyographic activity of human masticatory muscles. Am J Orthod Dentofacial Orthop 118: 328-324. [crossref]

5. Castelo PM, Gavião MB, Pereira LJ, Bonjardim LR (2007) Masticatory muscle thickness, bite force, and occlusal contacts in young children with unilateral posterior crossbite. Eur J Orthod 29: 149-156. [crossref]

6. Raustia AM, Salonen MA (1997) Gonial angles and condylar and ramus height of the mandible in complete denture wearers--a panoramic radiograph study. J Oral Rehabil 24: 512-516.[crossref]

7. Bonakdarchian M, Askari N, Askari M (2009) Effect of face form on maximal molar bite force with natural dentition. Arch Oral Biol 54: 201-204.[crossref]

8. Palinkas M, Nassar MS, Cecílio FA, Siéssere S, Semprini M, et al. (2010) Age and gender influence on maximal bite force and masticatory muscles thickness. Arch Oral Biol 55: 797-802.[crossref]

9. KoÃß D1, DoÄŸan A, Bek B (2011) Effect of gender, facial dimensions, body mass index and type of functional occlusion on bite force. J Appl Oral Sci 19: 274-279. [crossref]

10. de Abreu RA, Pereira MD, Furtado F, Prado GP, Mestriner Jr W, Ferreira LM (2014) Masticatory efficiency and bite force in individuals with normal occlusion. Arch Oral Biol 59: 1065-1074.[crossref]

11. Takaki P, Vieira M, Bommarito S (2014) Maximum bite force analysis in different age groups. Int Arch Otorhinolaryngol 18: 272-276.[crossref]
12. Roldán SI, Restrepo LG, Isaza JF, Vélez LG, Buschang PH (2016) Are maximum bite forces of subjects 7 to 17 years of age related to malocclusion? Angle Orthod 86: 456461.[crossref]

13. van der Bilt A, Tekamp A, van der Glas H, Abbink J (2008) Bite force and electromyograpy during maximum unilateral and bilateral clenching. Eur J Oral Sci 116: 217-222.[crossref]

14. Andrade Ada S, Gameiro GH, Derossi M, Gavião MB (2009) Posterior crossbite and functional changes. A systematic review. Angle Orthod 79: 380-386.[crossref]

15. Castelo PM, Bonjardim LR, Pereira LJ, Gavião MB (2008) Facial dimensions, bite force and masticatory muscle thickness in preschool children with functional posterior crossbite. Braz Oral Res 22: 48-54. [crossref]

16. Castelo PM, Pereira LJ, Bonjardim LR, Gavião MB (2010) Changes in bite force, masticatory muscle thickness, and facial morphology between primary and mixed dentition in preschool children with normal occlusion. Ann Anat 192: 23-26.[crossref]

17. Sonnesen L, Bakke M (2007) Bite force in children with unilateral crossbite before and after orthodontic treatment - A prospective longitudinal study. Eur J Orthod 29:310 313 .

18. Sonnesen L, Bakke M, Solow B (2001) Bite force in pre-orthodontic children with unilateral crossbite. Eur J Orthod 23: 741-749. [crossref]

19. Choi TH, Kim BI, Chung CJ, Kim HJ, Baik HS, et al. (2015) Assessment of masticatory function in patients with non-sagittal occlusal discrepancies. J Oral Rehabil 42: 2-9. [crossref]

20. YanikoÄŸlu N, Yilmaz B (2008) Radiological evaluation of changes in the gonial angle after teeth extraction and wearing of dentures: a 3-year longitudinal study. Oral Surg Oral Med Oral Pathol Oral Radiol Endod 105: e55-60.[crossref]

21. Rando C, Hillson S, Antoine D (2014) Changes in mandibular dimensions during the mediaeval to post-mediaeval transition in London: a possible response to decreased masticatory load. Arch Oral Biol 59: 73-81.[crossref]

22. Yoon SS, Chung CH (2015) Comparison of craniofacial growth of untreated Class I and Class II girls from ages 9 to 18 years: a longitudinal study. Am J Orthod Dentofacial Orthop 147: 190-196.[crossref]

23. Oksayan R, Aktan AM, Sökücü O, Hastar E, Ciftci ME (2012) Does the panoramic radiography have the power to identify the gonial angle in orthodontics? Scientific World Journal.

24. Shahabi M, Ramazanzadeh BA, Mokhber N (2009) Comparison between the externa gonial angle in panoramic radiographs and lateral cephalograms of adult patients with Class I malocclusion. J Oral Sci 51: 425-429. [crossref]

25. Zangouei-Booshehri M, Aghili HA, Abasi M, Ezoddini-Ardakani F (2012) Agreemen between panoramic and lateral cephalometric radiographs for measuring the gonial angle. Iran J Radiol 9: 178-182. [crossref]

26. El-Dawlatly MM, Fayed MM, Mostafa YA (2012) Deep overbite malocclusion analysis of the underlying components. Am J Orthod Dentofacial Orthop 142: 473 480. [crossref]

27. Antonarakis GS, Kjellberg H, Kiliaridis S (2013) Bite force and its association with stability following Class II/1 functional appliance treatment. Eur J Orthod 35: 434441. [crossref]

28. Xie QF, Ainamo A (2004) Correlation of gonial angle size with cortical thickness, height of the mandibular residual body, and duration of edentulism. J Prosthet Dent 91: 477-482.[crossref]

29. Joo JK, Lim YJ, Kwon HB, Ahn SJ (2013) Panoramic radiographic evaluation of the mandibular morphological changes in elderly dentate and edentulous subjects. Acta Odontol Scand 71: 357-362. [crossref]

30. Gregor C, Hietschold V, Harzer W (2013) A ${ }^{31}$ P-magnet resonance spectroscopy study on the metabolism of human masseter in individuals with different vertical facial pattern. Oral Surg Oral Med Oral Pathol Oral Radiol 115: 406-414. [crossref]

Copyright: (C2017 Said AV. This is an open-access article distributed under the terms of the Creative Commons Attribution License, which permits unrestricted use, distribution, and reproduction in any medium, provided the original author and source are credited. 